\title{
WiB - a new system concept for digital terrestrial television (DTT)
}

\author{
E. Stare1", J.J. Giménez², , P. Klenner ${ }^{3,}$ \\ ${ }^{1}$ Teracom, Sweden (erik.stare@teracom.se) \\ ${ }^{2}$ Universitat Politècnica de València, Spain (jorgigan@iteam.upv.es) \\ ${ }^{3}$ Panasonic Europe Ltd, Germany (peter.klenner@eu.panasonic.com)
}

\begin{abstract}
A new system concept for DTT, called "WiB", is presented, where potentially all frequencies within the Ultra High Frequency (UHF) band are used on all transmitter (TX) sites (i.e. reuse-1). The interference, especially from neighbouring transmitters operating on the same frequency while transmitting different information, is handled by a combination of a robust transmission mode, directional discrimination of the receiving antenna and interference cancellation methods. With this approach, DTT may be transmitted as a single wideband signal, covering potentially the entire UHF band, from a single wideband transmitter via the TX site. Thanks to a higher spectrum, utilisation, the approach allows for a dramatic reduction in fundamental power/cost and approximately a 37-60\% capacity increase for the same coverage as with current DTT. High speed mobile reception as well as fine granularity local services would also be supported, without any loss of capacity. The paper also outlines further possible developments of WiB, e.g. doubling the capacity via cross-polar Multiple In Multiple Out (MIMO), backward-compatible with existing receiving antennas, and adding a second, WiB-mobile, Layer Division Multiplexing (LDM) layer within the same spectrum, either as a mobile broadcast or as a mobile broadband.
\end{abstract}

\section{Introduction}

\section{Basic principles of WiB}

$\mathrm{WiB}$ is a new wideband reuse-1 based DTT concept, developed at Teracom, Sweden, by E. Stare. WiB builds on the earlier work of Cloud Transmission by $\mathrm{Wu}$ et al. (1), and is radically different from conventional DTT and offers very attractive characteristics. In a traditional High Power High Tower (HPHT) DTT Multi-Frequency Network (MFN) or Single Frequency Network (SFN), a high capacity is typically transmitted per UHF channel, e.g. 33-40 Megabits per second (Mbps) with the Digital Video Broadcasting (DVB)-T2 standard (2). However, the high order modulation that is needed to carry the high capacity makes the signal sensitive to interference, which requires transmitters that operate on the same frequency to be positioned sufficiently far away and in a regular pattern. This way, their respective signals are attenuated so as not to cause harmful interference when they are received. When SFNs are used, the same principle of separation applies to groups of SFN transmitters. A consequence of this approach is therefore that only a fraction $(1: \mathrm{N})$ of the frequencies at a particular site are actually used, which is called reuse-N frequency planning (for DTT, N is typically in the range 4 to 7). Unfortunately, since required power, according to Shannon (3), fundamentally increases exponentially with capacity, high-power transmitters (TXs) are necessary.

With WiB, a far more power-efficient approach is employed, which is to use potentially all UHF channels from all TX sites (reuse-1) and spread out the transmitted power equally across these channels. This can be achieved potentially as a single wideband signal using a single TX, where the existing $0.2-0.4 \mathrm{MHz}(2.5-5 \%)$ spectral gaps between UHF channels could also be exploited. Using reuse-1 and e.g. Quadrature Phase Shift Keying (QPSK) code rate at half the modulation allows for mobility and a spectral efficiency of about $1 \mathrm{bps} / \mathrm{Hz}$. This is about the same as with an "all DVB-T2" implementation of DTT using the existing type of frequency planning/reuse (assuming $5 \times 40 \mathrm{Mbps}$ MFN or $6 \times 33 \mathrm{Mbps}$ SFN). In both cases (taking overhead into account), approximately $200 \mathrm{Mbps}$ can be offered within the $224 \mathrm{MHz}$ of the DTT spectrum (470-694 MHz) that remains after the $700 \mathrm{MHz}$-band release. Simulations, however, indicate that $\mathrm{WiB}$ could be used with significantly higher spectral efficiency than $1 \mathrm{bps} / \mathrm{Hz}$ (1.37 to 1.60; see chapter about performance results below). The use of a very robust mode may also eliminate the need to use a Guard Interval (GI) altogether, since at low $\mathrm{C} / \mathrm{N}$ levels, the gain of using a GI seems to be lower than the overhead "pain".

A commonly employed DVB-T2 mode is 256-Quadrature Amplitude Modulation (QAM) code rate 2/3. However, with a QPSK rate of half the required TX power (for a given coverage) is about 50 times $(17 \mathrm{~dB})$ lower than the $8 \mathrm{MHz}$ channel. The net effect of this is that a WiB signal would fundamentally only require about $10 \%$ of the total TX power (of all multiplexes) for DVB-T2, see Figure 1. There are, however, also other WiB gains that may further reduce the required power, see below.

\section{Peak service data rate and tuner bandwidth}

Since the basic coding/modulation is restricted with $\mathrm{WiB}$, due to the reuse-1, the capacity within a single UHF channel will be limited, in the order of 7-10 Mbps. To compensate for the lower capacity within a particular UHF channel, it is instead assumed that a service can be spread across several UHF channels. Assuming the basic tuner bandwidth is increased by, for example, a factor of four, i.e. from one UHF channel $(8 \mathrm{MHz})$ to four UHF channels $(32 \mathrm{MHz})$, this would allow the peak data rate to be increased by a factor of four to around $28-40 \mathrm{Mbps}$ within this wider bandwidth. A side effect of the increased tuner bandwidth is an increased frequency diversity, which generally improves performance, assuming the service is appropriately interleaved across the entire bandwidth. This effect can be maximised by interleaving a service across the total used spectrum, e.g. by using frequency-hopping techniques like Time-Frequency Slicing (TFS), see (2) and Giménez et al. (4).

\section{Interference considerations}

With reuse-1, the receiver will experience a much lower Carrier-to-Interference ratio $(\mathrm{C} / \mathrm{I})$ than is usual for DTT and this issue must, of course, be seriously considered. The first tool to handle interference is the robust mode (e.g. $17 \mathrm{~dB}$ is more robust than current DTT), which may allow a C/I close to $0 \mathrm{~dB}$. The 


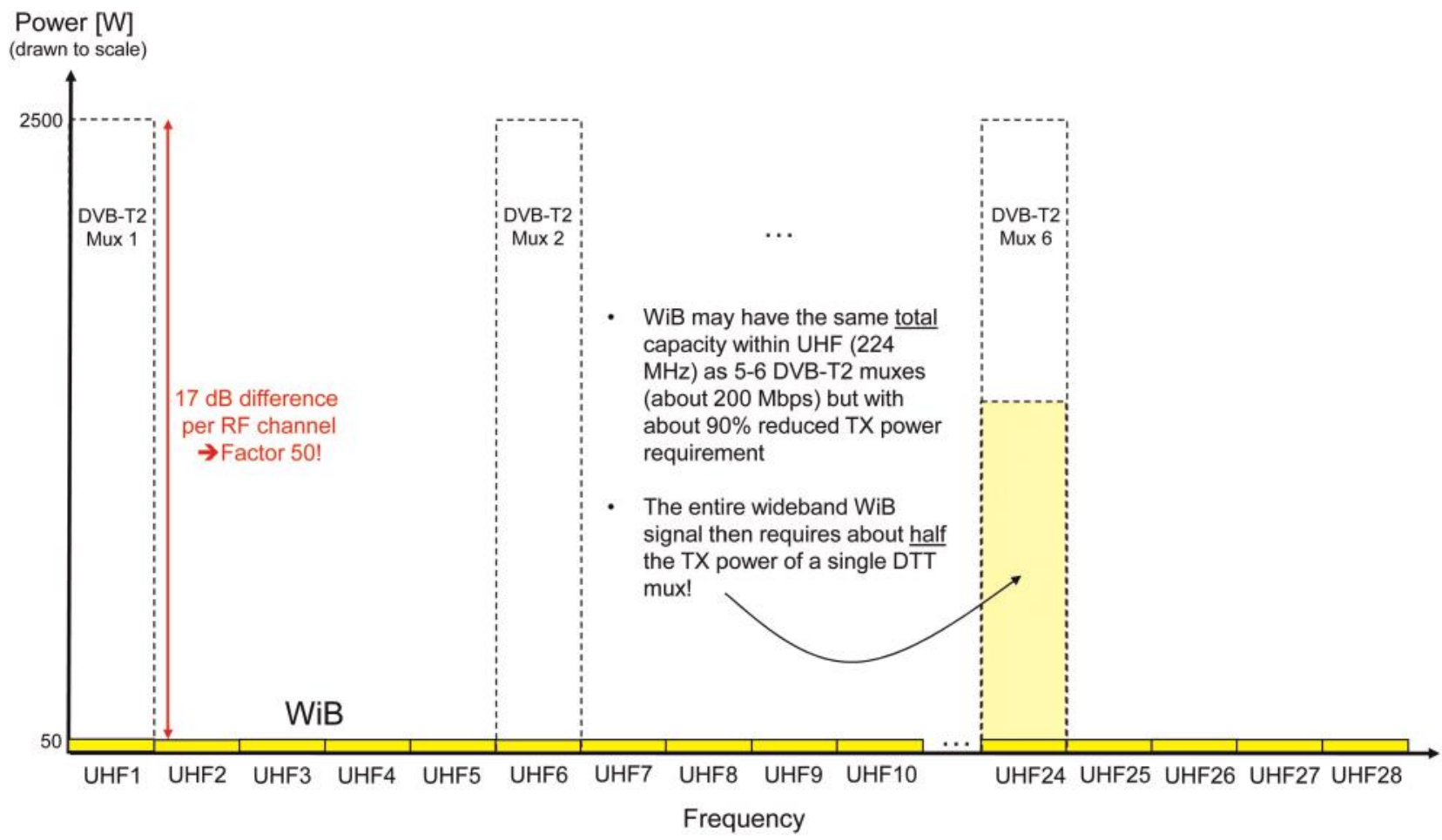

Figure 1 Required power for $D V B-T 2$ and $\mathrm{WiB}$

second tool (for fixed roof-top reception) is that a directional antenna typically offers a very significant (up to $16 \mathrm{~dB}$ ) discrimination, i.e. "attenuation" of signals in unwanted directions or polarisations. Finally, there are methods for interference cancellation, by which unwanted signals may be cancelled under certain conditions (see Interference Cancellation chapter below). It should be noted that thanks to the inherent ability to cope with interference from adjacent transmitters, the WiB concept allows potentially all TXs to transmit different content.

\section{Network cost savings with WiB}

\section{Savings in capital expenditures (CAPEX)}

Perhaps the most striking (CAPEX) cost saving is due to the fact that the total required TX power of the equipment could fundamentally be reduced by about $90 \%$, thereby considerably simplifying the infrastructure. This could allow all existing TXs to be replaced by a single wideband TX with a lower power (about 50\%) than each of the traditional DTT TXs. Some performance requirements, such as linearity, of the (single) TX could also be greatly relaxed thanks to the robust signal, which could simplify the design of the TX and also contribute to higher power efficiency. Furthermore, since the complete WiB signal can be transmitted as a single wideband signal, there is no need to use RF combiners anymore - there could be just a single exciter and a single wideband TX, with a Radio Frequency (RF) filter. A 90\% reduced power also reduces the cooling requirements very significantly and allows for simple battery back-up power solutions for many smaller sites.

Due to the lower power/cooling/volume/weight of the overall TX equipment, it could more easily be installed in the TX mast, which would also eliminate the need for RF feeders. WiB also lends itself well to be used together with "active TX antennas", i.e. a group of antenna elements (or even each antenna element) could potentially have its own very low power, wideband "mini-TX", which could enable an electronically-controlled phased array antenna, whereby the antenna diagrams could be tailor-made and optimised to the desired characteristics.

\section{Savings in operational expenditures (OPEX)}

Similar to the CAPEX case, the reduced power consumption would be the most striking OPEX advantage, but in this case, the cost reduction is seen in a reduced electricity bill. In addition to the fundamental $90 \%$ power reduction, there are also other possible factors that could allow for a further reduction, in consumed power, such as elimination of the attenuation in (now superfluous) combiners, feeder, RF split etc., which may amount to a total of about $3 \mathrm{~dB}$ and a consequent further power reduction of $50 \%$. On top of that, increased frequency diversity may offer further power consumption gains, due to a better link budget. Depending on the type of TX, there may also be power efficiency gains in the actual TX implementation due to lower linearity requirements. From a service perspective, the overall complexity of the system would be reduced since there are fewer system components and the sensitivity of these is also reduced thanks to a far more robust operation mode. Lower power may also generally increase transmitter lifetime and reduce failure probability. Furthermore, there will be a reduced or no need for frequency changes or frequency re-planning once the network is in operation.

\section{Interference cancellation with WiB}

\section{Layer division multiplexing-based interference cancellation (LDM-IC)}

When a target signal S1 is interfered by an equally-modulated but stronger S2 signal, LDM-IC (also referred to as Successive Interference Cancellation) can be implemented by first demodulating the stronger signal, then re-modulating it and subtracting it from the incoming signal, which is possible since it is perfectly known after the (assumed successful) demodulation. In a final step, the target $\mathrm{S} 1$ signal can then be demodulated. This process is possible as long as the actual Carrier-to-Noise +Interference ratio $(\mathrm{C} /(\mathrm{N}+\mathrm{I}))$ of the signal to be demodulated (here $\mathrm{S} 2$ followed by $\mathrm{S} 1)$ is larger than the required $\mathrm{C} /(\mathrm{N}+\mathrm{I})$. For a $1 \mathrm{bps} / \mathrm{Hz}$ spectral efficiency (e.g. half the QPSK rate), the required $\mathrm{C} /(\mathrm{N}+\mathrm{I})$ is close to $0 \mathrm{~dB}$. The described process can be generalised 

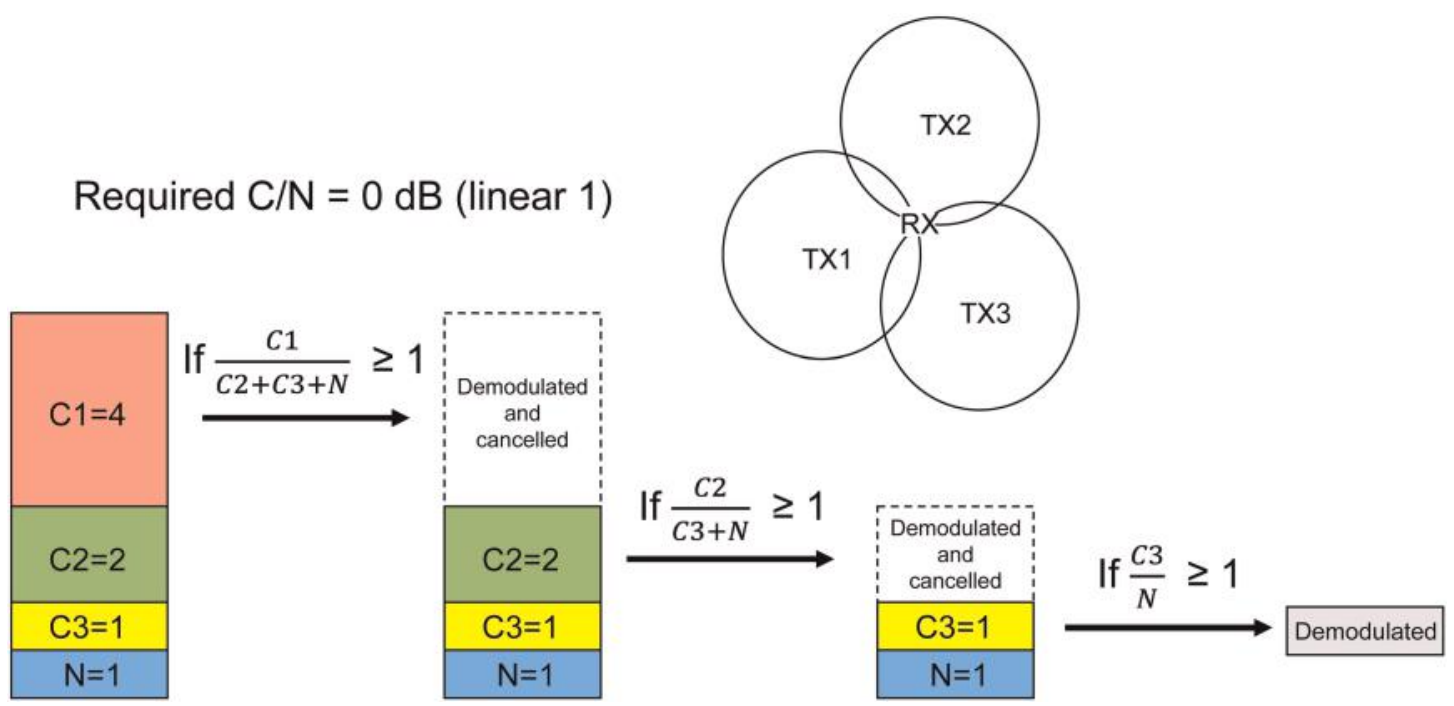

Figure 2 Example of LDM-IC, where the weakest TX3 signal (received with power C3) is demodulated in three steps

and used for any number of signals as long as the $\mathrm{C} /(\mathrm{N}+\mathrm{I})$ requirement is fulfilled for every demodulated and subtracted signal.

One example scenario is shown in Figure 2 above, where the received power from $\mathrm{TX} 1, \mathrm{TX} 2$ and $\mathrm{TX} 3$ are $\mathrm{C} 1, \mathrm{C} 2$ and $\mathrm{C} 3$ respectively, with $\mathrm{N}$ being noise power. The weakest TX3 signal can be demodulated as long as the $\mathrm{C} /(\mathrm{N}+\mathrm{I})$ is fulfilled in the cancellation process of the two stronger signals and the required $\mathrm{C} / \mathrm{N}$ is fulfilled for TX3.

For LDM-IC to work (with reasonable complexity) in WiB, all involved transmitted signals need to be fully synchronised with aligned Forward Error Correction (FEC) blocks, i.e. the reception situation needs to look similar to a traditional SFN, but with the different TXs transmitting different content. To allow for LDM-IC, each received TX also needs to include scattered pilots that are orthogonal to all other received TX signals involved in the interference cancellation, i.e. there needs to be at least three orthogonal pilot patterns.

\section{Antenna-based interference cancellation (ANT-IC)}

A completely different approach to interference cancellation is to use multiple receiving antennas, e.g. arranged as a phased-array antenna. Even in the simplest case, with two dipoles, these could have an electronically-controlled beam, which could dynamically maximise the $\mathrm{C} /(\mathrm{N}+\mathrm{I})$ of the signal to be received, such as by full cancellation of one signal.

\section{Combination of LDM-IC and ANT-IC}

The most powerful, approach for interference cancellation identified to date is to combine LDM-IC with ANT-IC in such a way that for each signal to be demodulated, the $\mathrm{C} /(\mathrm{N}+\mathrm{I})$ is maximised by appropriately adjusting/optimising the electronically-controlled antenna for this particular signal. When this signal has been demodulated/cancelled, the antenna may be retuned and the $\mathrm{C} /(\mathrm{N}+\mathrm{I})$ can again be maximised for the next signal to be demodulated.

\section{Variable bit rate (STATMUXED) services using multiple PLPs}

Variable bit rate services may be transmitted over DVB-T2 using variable bit rate Physical Layer Pipes (PLPs). However, with WiB LDM-IC would not generally work with variable bit rate PLPs, since the signals from the associated TXs (varying independently) would then not be synchronised as required. With WiB, all PLPs have an equal and constant bit rate instead, and the VBR aspect is catered for by dynamically mapping a VBR service to a variable number of PLPs; the number being dependent on the instantaneously required bit rate of the service. The efficiency of the statistical multiplexing would not be significantly affected by this.

\section{Performance results from simulations}

\section{Spectral efficiency limits with HPHT network modelling}

The achievable spectral efficiency of WiB in a HPHT network was estimated via Monte Carlo simulations. The network was modelled by a homogeneous reuse-1 hexagon lattice, see Figure 3, with 60 $\mathrm{km}$ distance between adjacent TXs and with an effective antenna height of $250 \mathrm{~m}$. Spectral efficiency was evaluated at the assumed worst point of the network, i.e. the mid-point between three

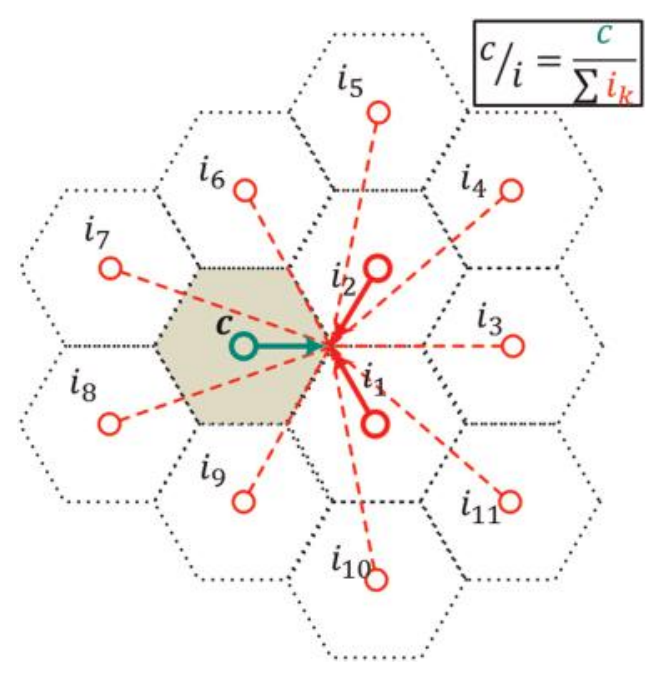

Figure 3 Hexagon lattice

Table 1 WiB spectral efficiency

\begin{tabular}{lcc}
\hline Time correlation type & Best TX & Wanted TX \\
\hline Inter/Intra site (C) & $3.41 \mathrm{bps} / \mathrm{Hz}$ & $1.55 \mathrm{bps} / \mathrm{Hz}$ \\
Intra-site (U1) & $3.38 \mathrm{bps} / \mathrm{Hz}$ & $1.37 \mathrm{bps} / \mathrm{Hz}$ \\
No correlation (U2) & $4.07 \mathrm{bps} / \mathrm{Hz}$ & $1.60 \mathrm{bps} / \mathrm{Hz}$ \\
\hline
\end{tabular}




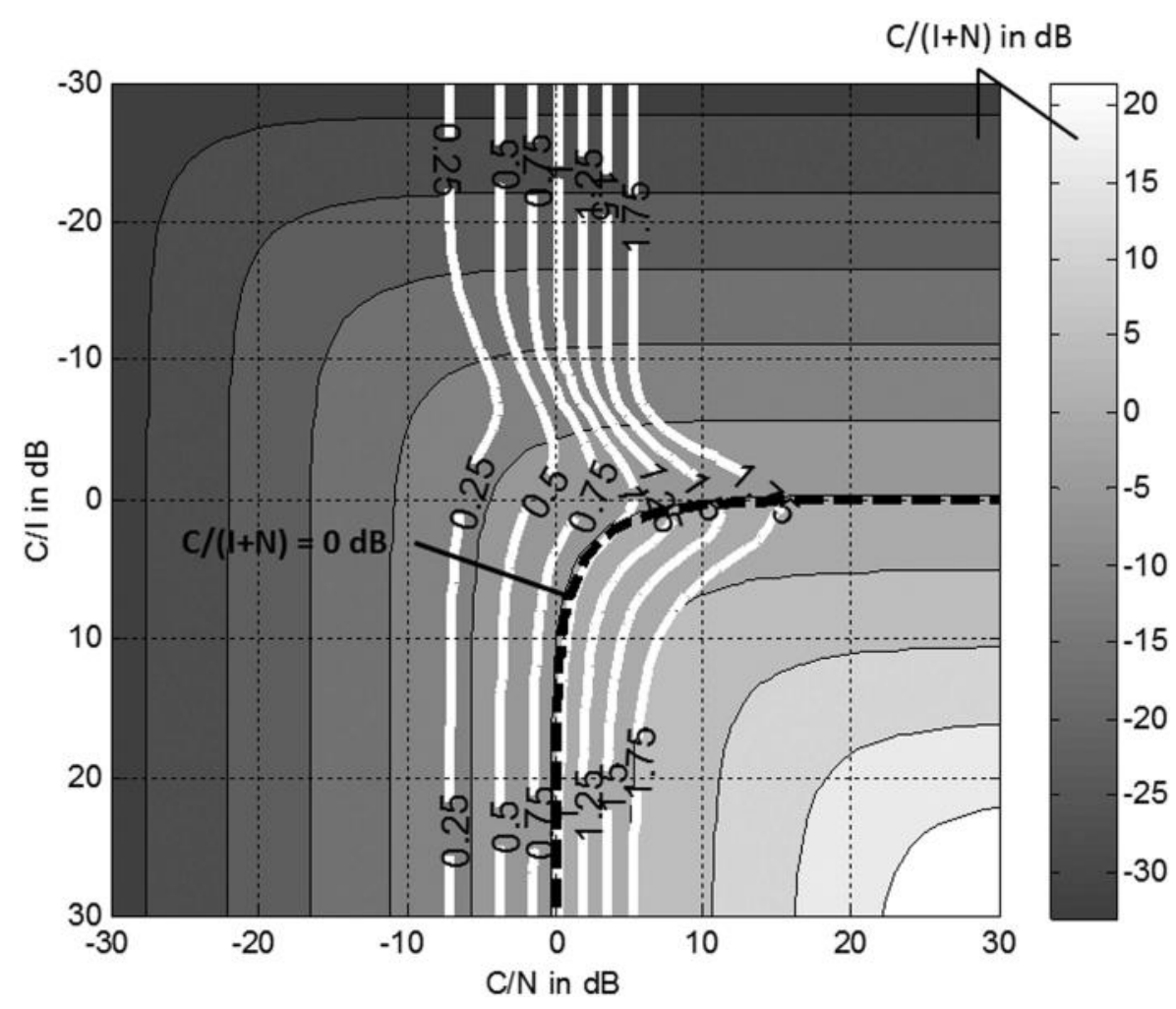

Figure 4 Achievable rates $0.25 \ldots 1.75 \mathrm{bps} / \mathrm{Hz}$ for a two-TX system with an applied random phase and optimal detection

adjacent TXs, at $10 \mathrm{~m}$ above ground level, see Figure 3. The assumed Effective Radiated Power (ERP) was $1 \mathrm{~kW}$ per $8 \mathrm{MHz}$ UHF channel and the receiving antenna gain was $11 \mathrm{dBd}$, with discrimination according to International Telecommunication Union (ITU) Rec. BT.419 (5). The assumed down-lead loss and receiver noise figure were $4 \mathrm{~dB}$ and $6 \mathrm{~dB}$ respectively. The propagation model was according to ITU Recommendation ITU-R P.1546 (6) over land. Time variations of the propagation was statistically modelled by fitting two log-normal distributions to the propagation curves given for $50 \%$ and $10 \%$, and $10 \%$ and $1 \%$ of time, respectively. This allowed three different correlation models to be used: full inter-site as well as intra-site correlation (C), no inter-site but full intra-site correlation (U1), no inter- or intra-site correlation (U2). Fading was statistically modelled by means of

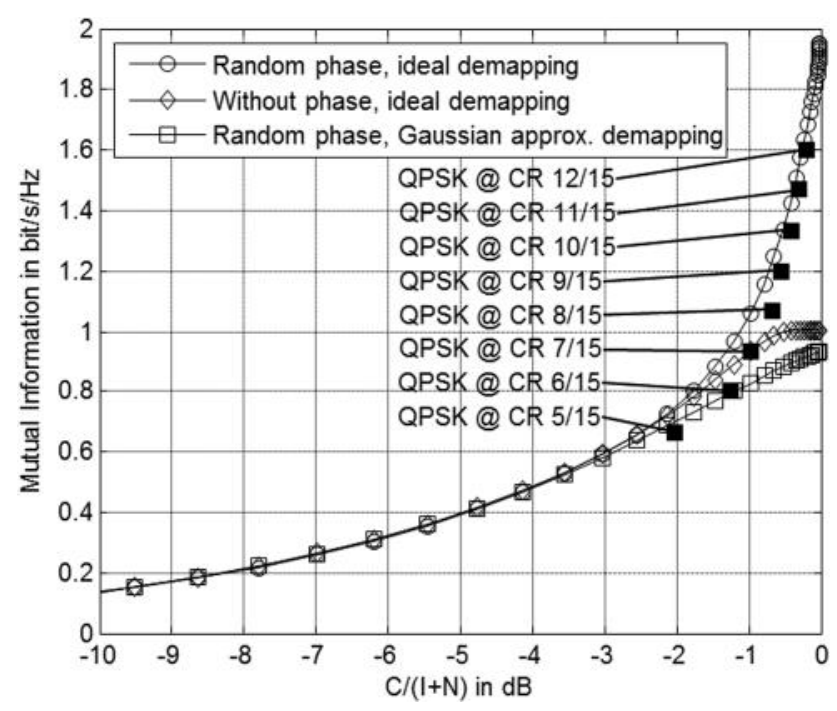

Figure 5 For $C / I=0 d B$, achievable rates for various de-mappers. Included is the performance with ATSC $3.0 \mathrm{CRs}$ ( two processes. A frequency-independent but location-dependent fading was modelled as a log-normal random variable with $0 \mathrm{~dB}$ mean and $5.5 \mathrm{~dB}$ standard deviation. A site-to-site correlation model is applied to account for location-dependent fading on angular position and distance between stations (7). A frequency dependent fading process with $2 \mathrm{~dB}$ standard deviation was added to model potential frequency-dependent variations of the received field strength, according to Giménez et al. (8). The coverage criterion was to maintain a reception with $95 \%$ location probability and for $99 \%$ of the time at the worst point in the network. For each realisation, the $\mathrm{C} /(\mathrm{N}+\mathrm{I})$ determined the maximum Shannon capacity that can be transmitted. In order to account for ideal frequency interleaving, the average spectral efficiency over all RF channels was calculated.

Two application cases were considered. Best $T X$ models a receiver pointing to the best TX in each realisation. Wanted $T X$ models a receiver pointing to a desired (not necessarily the best) TX among the three closest transmitters. The spectral efficiency that the WiB system can provide is calculated so that layers above the desired one can be cancelled. Since all layers provide the same capacity, the minimum of all of them is selected. Table 1 shows the achievable spectral efficiency with $95 \%$ location probability for $99 \%$ of time.

\section{Optimised performance for QPSK interfered by QPSK}

In the HPHT network simulations above, all interfering signals that are weaker than the one currently being demodulated have been treated as noise. However, this is a pessimistic assumption since the constellation is known, and this a priori information can be exploited to improve performance. From an information-theoretical point of view, based on the concept of Mutual Information, one can derive the theoretically optimum performance for a general case where a QPSK signal suffers interfered by another QPSK signal, having an added random phase. The random phase will usually arise naturally as a result of different path delays in the network combined with interleaving, but can be added intentionally at the transmitter to avoid a destructive superposition 


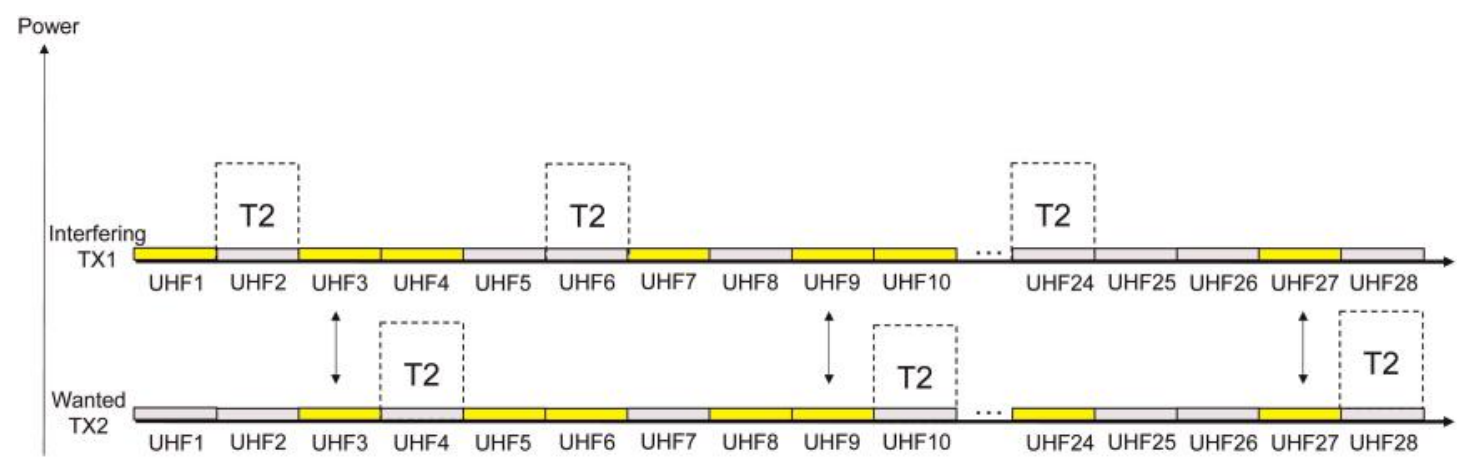

Figure 6 WiB interleaved with DTT

of cells at the receiver under highly correlated Line-of-Sight (LoS) propagation conditions. This has been done and the required $\mathrm{C} / \mathrm{N}$ for a given power relation of the two signals $(\mathrm{C} / \mathrm{I})$ is given in Figure 4 for a number of different spectral efficiencies $(\mathrm{bps} / \mathrm{Hz}$ ). For $1 \mathrm{bps} / \mathrm{Hz}$, with required $\mathrm{C} / \mathrm{N}=0 \mathrm{~dB}$, adding a noise-like interferer at $\mathrm{C} / \mathrm{I}=0 \mathrm{~dB}$ would leave no room for noise thus causing the required $\mathrm{C} / \mathrm{N}$ to approach infinity. However, when the QPSK constellation is taken into account in an optimum way (ideal demapping) with 2-dimensional log-likelihood ratios (2D-LLRs), the required $\mathrm{C} / \mathrm{N}$ becomes $6.0 \mathrm{~dB}$. Exploiting the knowledge of the constellation therefore seems to allow very large gains in performance. Network performance is expected to be further improved when this behaviour is exploited. Figure 5 depicts the achievable rates for a fixed $\mathrm{C} / \mathrm{I}=0 \mathrm{~dB}$ assuming optimal and suboptimal de-mapping. Depicted are also rates for Advanced Television Systems Committee (ATSC) 3.0 (9) with QPSK and different code rates (CRs) based on the presence of a random phase, optimal de-mapping and sum-product FEC decoding. As can be seen, ATSC codes closely follow the ideal curve.

\section{Receiver aspects}

The receiver could basically be a single-tuner Orthogonal Frequency Division Multiplex (OFDM) receiver (or other Multi-Carrier system) with at least a $32 \mathrm{MHz}$ tuner bandwidth. The wider virtual bandwidth (up to e.g. $224 \mathrm{MHz}$ ) is either handled by TFS (frequency hopping) or by sampling at a higher rate and then performing the frequency hopping in the digital domain. To maintain the same OFDM symbol time and carrier spacing in this $32 \mathrm{MHz}$ bandwidth as with the DVB-T2 $32 \mathrm{~K}$ mode in $8 \mathrm{MHz}$, a Fast Fourier Transform (FFT) size of $2^{17}(128 \mathrm{~K})$ would be required. For channel estimation, three different channel estimates (one for each received TX signal in a 3-TX environment) need to be handled and passed through the (cell-based) de-interleaving chain, so that the LDM-IC process can be performed after a single "once-and-for-all" de-interleaving step. The complexity of the fundamental FEC-decoding itself could be similar to DVB-T2 or ATSC 3.0 (or maybe lower, because of the lower expected peak bit rate), but the interference cancellation would require the basic FEC decoding to be perfomed at a higher rate (or with more parallelism).

Considering that Moore's Law has been in action since the specification and implementation of DVB-T2 in 2009 and is expected to be in operation until the implementation of WiB sometime in the 2020 's, the overall $\mathrm{WiB}$ receiver complexity does not appear, in fact, to be overly complex.

\section{Introduction scenarios}

All migrations to new standards are more or less difficult and can normally not be achieved in "one shot". Instead, a gradual approach is typically preferred. Two different approaches have been identified to introduce WiB: "Dedicated band" or "Interleaved". With the dedicated-band approach, part of the UHF band would be released for $\mathrm{WiB}$ in a similar way as the $700 \mathrm{MHz}$ band will be released, with similar international co-ordination. A contiguous sub-part of the UHF band could then be used for WiB, ideally with a synchronised introduction. In later steps, this band could potentially be extended to the full UHF band. With the interleaved approach, WiB would be introduced in much the same way as DTT was introduced in an analogue TV context, i.e. current DTT would still be used and WiB would be transmitted with some power from potentially all frequencies that are not used by current DTT. The transmitted WiB power would be adjusted for each TX site/frequency individually, so as not to cause harmful interference. In a transition phase, it might be necessary to accept some degree of degradation of the existing DTT, although this may also be compensated for by using a somewhat more robust transmission mode for DTT. It is possible to design WiB to allow the use of an arbitrary subset of UHF channels (with the particular subset varying across TX sites), still allowing for interference cancellation, see Figure 6. This subset could first be small and then grow until WiB eventually might replace DVB-T/T2 altogether.

\section{Further work, advanced WiB}

The basic WiB system outlined earlier in this paper could be further developed in a number of different ways. Here we will only mention two such directions, both of which could double the WiB capacity: WiB-MIMO and WiB-LDM. With WiB-MIMO, one would add a second independent signal on the opposite polarisation and effectively have a dual-Single Input Single Output (SISO) signal, where one of the signals would be receivable by a legacy single-polarisation TV antenna, thanks to antenna polarisation discrimination. By frequency-transposing one of the two received polarised components immediately after the antenna, the existing down-lead could be used for both components and the receiver could be an SISO receiver, which would choose a selected service from the relevant component. With WiB-LDM, one would add a second WiB-mobile, LDM layer superimposed on the WiB-DTT signal, and transmit this from the TXs of a densified network providing mobile coverage of the WiB-Mobile signal. The weaker WiB-DTT signal would still be strong enough for rooftop reception after cancellation of the stronger WiB-Mobile signal. In one "fully converged" scenario, the WiB-Mobile signal could even be a mobile broadband (bi-directional unicast) signal, e.g. as part of $5 G$ New Radio.

\section{Conclusions}

This paper has presented a new DTT system concept called WiB, based on Wideband reuse-1, which allows for a potentially very large reduction of DTT network costs (both CAPEX and OPEX) while significantly exceeding the capacity and coverage that can be obtained with an optimised implementation of DVB-T2, for example. In the most demanding "Wanted TX" case, simulations 
indicate a possible capacity increase in the range of $37-60 \%$ assuming the DVB-T2 reference can carry about $200 \mathrm{Mbps}$ per site within the $470-694 \mathrm{MHz}$ band. The complexity of the receiver could be limited to that required to receive a particular service and not the entire transmitted WiB capacity. Furthermore, the WiB concept also allows for high-speed mobile reception, without the need for handover or capacity loss, fine content granularity (no big SFN areas required) and a possible reduction (or even elimination) in GI overhead. Finally, WiB can be extended in various ways, such as backward-compatible cross-polar MIMO, dual-layer LDM mobile/fixed reception and even with a WiB-DTT and WiBmobile broadband sharing literally the same spectrum.

\section{Acknowledgments}

The authors would like to thank Oliver Haffenden (BBC) for valuable comments. Erik Stare would also like to thank Magnus Ahxner and Staffan Bergsmark (both at Teracom) for valuable discussions and inspiration.

\section{References}

1 Wu, Y, Rong, B, Salehian, K, Gagnon, G.: 'Cloud transmission: a new spectrum-reuse friendly digital terrestrial broadcasting transmission system', IEEE Trans. Broadcast., September 2012, 58, (3), pp. 329-337

2 ETSI EN 302 755: 'Digital video broadcasting (DVB); frame structure channel coding and modulation for a second generation digital terrestrial broadcasting system (DVB-T2)'

3 Shannon, C.A.: 'A mathematical theory of communication', Bell Syst. Tech. J., July, October, 1948, 27, pp. 379-423, 623-656

4 Giménez, J, Stare, E, Bergsmark, S, Gómez-Barquero, D.: 'Time frequency slicing for future digital terrestrial broadcasting networks', IEEE Trans. Broadcast., June 2014, 60, (2), pp. 227-238

5 ITU Recommendation ITU-R BT.419-3: 'Directivity and polarization discrimination of antennas in the reception of television broadcasting', 1992

6 ITU Recommendation ITU-R P.1546-5: 'Method for point-to-area predictions for terrestrial services in the frequency range $30 \mathrm{MHz}$ to $3000 \mathrm{MHz}, 2013$

7 Saunders, S, Zavala, A.A.: 'Antennas and propagation for wireless communication systems' (Wiley, New York, NY, USA, 2007, 2nd edn.)

8 Giménez, J, Gozálvez, D, Gómez-Barquero, D, Cardona, A.: 'A statistical model of the signal strength imbalance between RF channels in a DTT network', Electron. Lett., 7th June 2012, 48, (12)

9 ATSC 3.0 Candidate Standard: 'Physical Layer Protocol (A/322)', available at: www.atsc.org 